\title{
Central facial weakness due to medial medullary infarction: the course of facial corticobulbar fibres
}

\author{
S Terao, S Takatsu, M Izumi, J Takagi, T Mitsuma, A Takahashi, A Takeda, G Sobue
}

Division of Neurology, Fourth Department of Internal Medicine, Aichi Medical University, Nagakute, Aichi, Japan

$S$ Terao

S Takatsu

M Izumi

J Takagi

T Mitsuma

Tokai Central Hospital, Kakamigahara, Gifu, Japan

A Takahashi

Department of Neurology, Chubu National Hospital, Obu, Aichi, Japan A Takeda

Department of Neurology, Nagoya University School of Medicine, Nagoya, Japan

G Sobue

Correspondence to: Dr Shin-ichi Terao, Division of Neurology, Fourth Department of Internal Medicine, Aichi Medical University, Nagakute, Aichi 480-11, Japan.

Received 21 January 1997 and in revised form 25 March 1997

Accepted 27 March 1997

\begin{abstract}
Two patients are reported with contralateral hemiparesis including a face of supranuclear type, caused by an infarct of the unilateral ventromedial part of the upper medulla. Data from these patients support the hypothesis that part of the corticobulbar fibres supplying the lower facial muscles descend ipsilaterally in the ventromedial part of the upper medulla and then, after decussation, ascend rostrally to the contralateral facial nucleus.
\end{abstract}

(F Neurol Neurosurg Psychiatry 1997;63:391-393)

Keywords: facial nerve; corticobulbar tract; brain stem infarction; magnetic resonance imaging

The anatomical distribution and the function of human peripheral facial nerves are well known, but little is known of the course of the corticobulbar fibres that connect the motor cortex with the facial nucleus. ${ }^{1-3}$ The facial corticobulbar fibres provide strongly unilateral innervation to the contralateral lower facial muscle and bilateral innervation to the upper facial muscles. ${ }^{1-3}$ Classic symptomatic localisation postulates that lesions rostral to the upper mid-pons result in contralateral facial weakness of a central type, whereas ipsilateral facial weakness of the peripheral type ensues from lesions involving the inferolateral portion of the pons. $^{34}$ We report two patients in whom contralateral lower facial weakness of central type, in addition to contralateral hemiparesis of the limbs, resulted from unilateral medial medullary infarction.

\section{Case reports}

PATIENT 1

A 40 year old man presented to our hospital with a weakness of the right limbs and dysarthria which began while he was driving a car five days previously. At the age of 30 he was noted to have hypertension. On admission he was alert, mildly obese, with a blood pressure of $134 / 80 \mathrm{~mm} \mathrm{Hg}$ and a regular heart rate of 70 beats/min. Neurologically, there were no abnormalities of eye movement or pupillary function, and the strength of the frontalis and orbicularis oculi muscles was well preserved. However, he had right lower facial weakness and mild flattening of the right nasolabial fold. There was weakness of the left half of the tongue. Slight paralytic dysarthria was also present, although dysgeusia, paresis of the soft palate, sensory impairment of the face, hypoacousis, and dysphagia were absent. Right hemiparesis with prominent weakness of the arm was noted. Deep tendon reflexes were exaggerated in the right limbs and Babinski's sign was present on the right side. Light touch and vibratory sensation were slightly diminished in the right leg, whereas temperature and pain sensation were well preserved. Transthoracic echocardiogram, ECG, and Holter monitoring findings were normal. Brain MRI performed on day 10 after onset of disease disclosed a small area of infarction in the left ventromedial part of the upper medulla (fig 1A) and no other lacunar infarctions of the deep white matter. Cerebral angiography at 12 days post-onset showed mild atherosclerotic changes of the vertebrobasilar arteries and non-visualisation of the anterior spinal artery. The right vertebral artery exhibited hypoplastic occlusion. Although the hemiparesis of the right arm remained, the right facial weakness and left tongue paralysis improved gradually and resolved within one month of onset.

\section{PATIENT 2}

A 65 year old man was admitted to our hospital after the onset of weakness in his right leg two days earlier, with consequent difficulty in walking. $\mathrm{He}$ was noted to have diabetes mellitus and hypertension two years previously, and had been on an antihypertensive drug. On admission he was alert and had a blood pressure of $154 / 72 \mathrm{~mm} \mathrm{Hg}$ with no arrhythmia. Neurologically, there were no abnormalities of eye movement or pupillary function. The strength of the frontalis and orbicularis oculi muscles was well preserved, but he had a right lower facial weakness with mild flattening of the right nasolabial fold and paralytic dysarthria. There was no paralysis of the tongue nor other cranial nerve deficit. He exhibited a severe right hemiparesis and hyperreflexia with a Babinski's sign. Sensory testing was normal to all modalities. Transthoracic echocardio- 


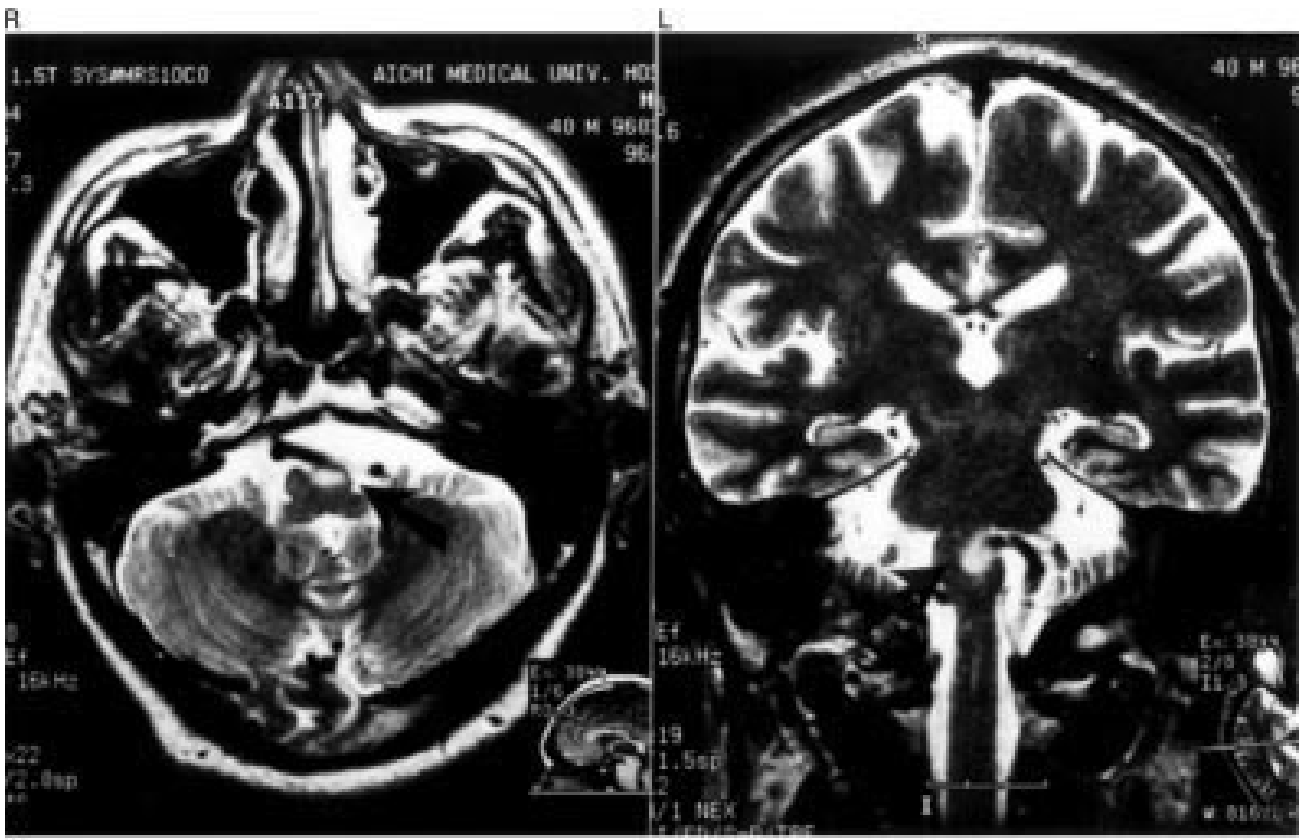

A

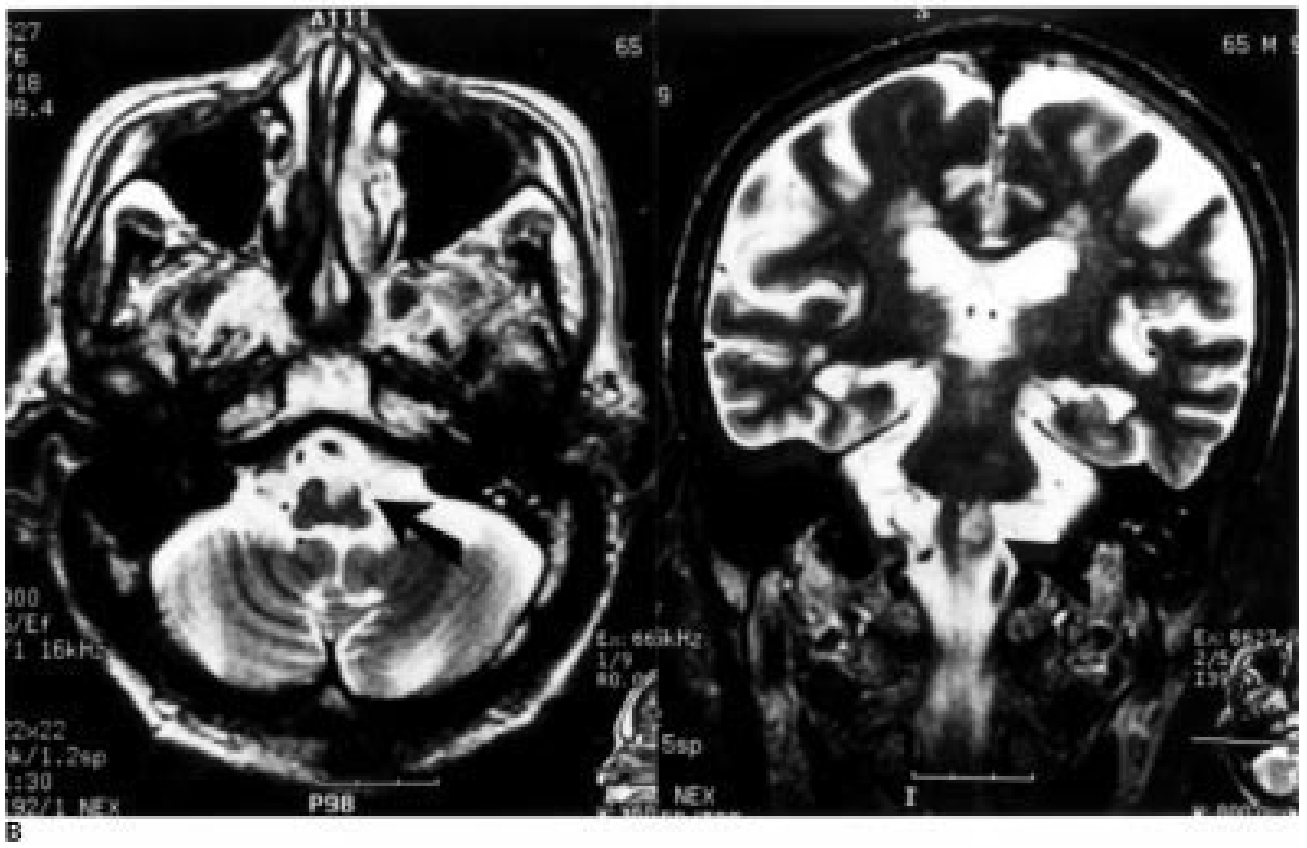

Figure 1 (A) Axial (left) and coronal (right) T2 weighted (5000/96, TR/TE) MRI of patient 1. A high signal intensity area (arrow) is present in the left ventromedial part of the upper medulla. (B) Axial (left) and coronal (right) T2 weighted (4000/96, TR/TE) MRI of patient 2. A high signal intensity area (arrow) is present in the left ventromedial part of the upper medulla.

gram, ECG, and Holter monitoring findings were all normal. Brain MRI performed 10 days after onset showed an infarct of the left ventromedial part of the upper medulla (fig 1B) and old multiple lacunar infarctions of the deep white matter of the cerebral hemispheres. Two weeks after onset MR angiography disclosed atheromatous changes involving the left vertebrobasilar arteries, although no apparent stenosis or occlusion was found. The right hemiparesis remained two months after the onset, yet the right facial weakness resolved within three weeks.

\section{Discussion}

Although contralateral facial weakness is usually caused by lesions rostral to the facial nucleus, it is important to realise that lesions caudal to the facial nucleus can also cause contralateral facial weakness.

There have also been several reports of mild facial weakness caused by medial medullary infarction, ${ }^{5-11}$ but there were few details on the responsible lesion. Currier ${ }^{6}$ hypothesised that the corticobulbar fibres to the lower facial nucleus leave the pyramid at the pontomedullary junction and descend caudally to at least 


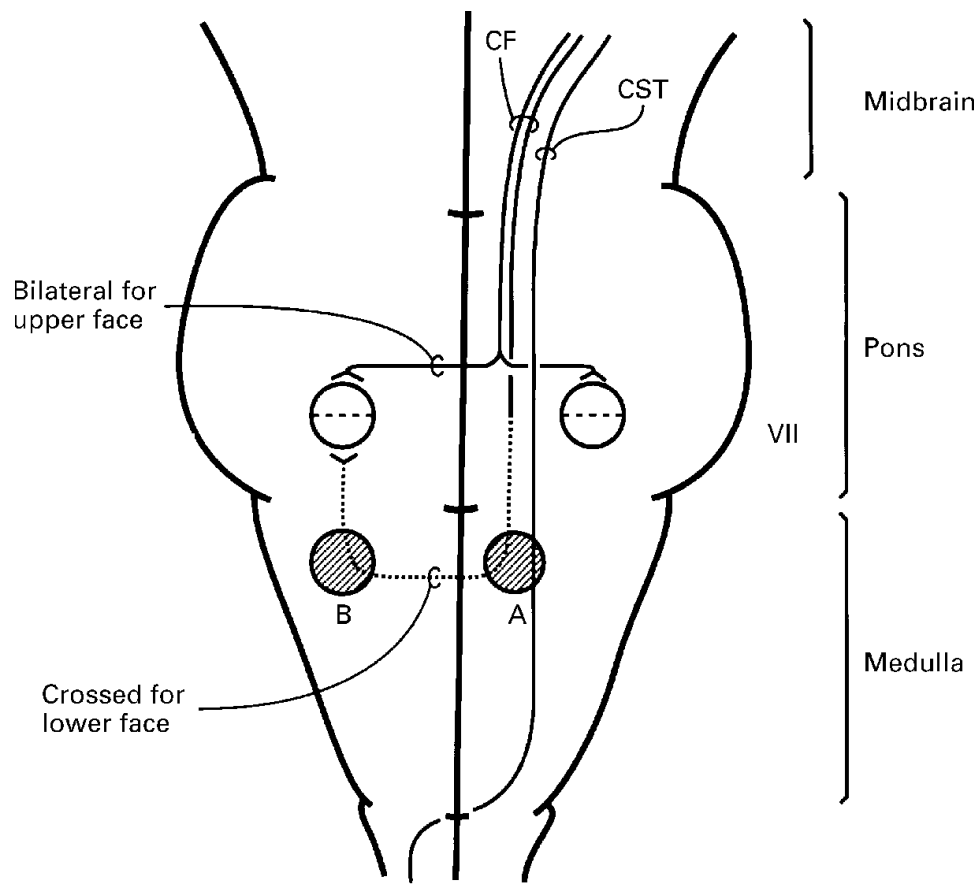

Figure 2 Schematic diagram of the pathways of the facial corticobulbar fibres. (A) (our patients) and (B) (lateral medullary infarction) show affected sites of the corticobulbar fibres. CST represents corticospinal tract; CF represents facial corticobulbar fibres.

mid-medullary levels, and then most fibres cross to the opposite facial nucleus. It has been postulated that interruption of these fibres by infarction at a predecussation level results in the contralateral central facial weakness. There have been some reports of facial weakness resulting from a similar mechanism by medial medullary infarction, ${ }^{7812} 13$ although it has been established pathologically that the facial nucleus received such corticobulbar fibres. ${ }^{14}$ Recently, Cavazos et $a l^{10}$ reported a patient with an infarction of the medullary pyramid with pure motor hemiparesis including the lower face. Based on the MRI findings, they hypothesised that some of the facial corticobulbar fibres descend ipsilaterally, making a loop as low as the upper medulla before decussating and ascending to the contralateral facial nucleus. We suggest that the findings in our present patients serve to support Cavazos's description on the course of the facial corticobulbar fibres.

The absence of facial weakness in several reported cases may be consistent with a more caudal medullary lesion. ${ }^{12}{ }^{15} 16$ On the other hand, lateral medullary infarction relatively often results in facial weakness on the side of the lesion. ${ }^{14}{ }^{17-20}$ It has also been postulated that facial weakness on the side of the lesion results from corticobulbar fibres being interrupted while they ascend contralaterally after decussating. ${ }^{13} 1720$

We have proposed a scheme for the facial corticobulbar fibres (fig 2). In our patients, left ventromedial lesion of the upper medulla may give rise to right central facial weakness because the corticobulbar fibres are affected at predecussation levels (fig 2A). On the other hand, the lateral medullary infarction is likely to result in ipsilateral central facial weakness because the corticobulbar fibres are interrupted at postdecussation levels (fig 2B). Therefore, it is suggested that part of the pathways of the facial corticobulbar fibres descend ipsilaterally adjacent to the ventromedial part of the corticospinal tract to the upper medulla before decussating, and ascend contralaterally in the dorsolateral part of the medulla to the facial nucleus.

MRI allows a clear delineation of lesions of the brain stem and spinal cord. It is hoped that further neuroanatomical study will clarify in detail the courses of central pathways including the corticobulbar and corticospinal tracts.

This work was supported in part by a grant from the Aichi Health Promotion Foundation and a grant from the Ministry of Health and Welfare of Japan.

1 Kuypers HGJM. Corticobulbar connexions to the pons and lower brain-stem in man. An anatomical study. Brain 1958;81:364-88.

2 Parent A. Pons. In: Parent A, ed. Carpenter's human neuroanatomy. 9th ed. Baltimore: Williams and Wilkins, 1996:469-526.

3 Patten J. The brain stem. In: Patten J, ed. Neurological differential diagnosis. 2nd ed. London: Springer, 1996:162-77.

4 Adams RD, Victor M. Motor paralysis. In: Adams RD, Victor M, Ropper AH, eds. Principles of neurology. 6 th ed. New tor M, Ropper AH, eds. Principles

5 Chokroverty S, Rubino FA, Haller C. Pure motor hemiplegia due to pyramidal infarction. Arch Neurol 1975;32:6478.

6 Currier RD. The medial medullary syndrome. Fournal of the University of Michigan Medical Centre 1976;42:96-104.

7 Ho K-L, Meyer KR. The medial medullary syndrome. Arch Neurol 1981;38:385-7.

8 Kase CS, Varakis JN, Stafford JR, et al. Medial medullary infarction from fibrocartilaginous embolism to the anterior infarction from fibrocartilaginous em

9 Kim JS, Kim HG, Chung CS. Medial medullary syndrome. Report of 18 new patients and a review of the literature. Stroke 1995;26:1548-52.

10 Cavazos JE, Bulsara K, Caress J, et al. Pure motor hemiplegia including the face induced by an infarct of the medullary pyramid. Clin Neurol Neurosurg 1996;98:21-3.

11 Toyoda K, Imamura T, Saku Y, et al. Medial medullary infarction: analyses of eleven patients. Neurology 1996;47: 1141-7.

12 Tyler KL, Sandberg E, Baum KF. Medial medullary syndrome and meningovascular syphilis: a case report in an HIV-infected man and a review of the literature. Neurology 1994;44:2231-5.

13 Gan R, Noronha A. The medullary vascular syndromes revisited. $\mathcal{F}$ Neurol 1995;242:195-202.

14 Fisher CM, Karnes WE, Kubik CS. Lateral medullary infarction- the pattern of vascular occlusion. $\mathcal{F}$ Neuropathol Exp Neurol 1961;20:323-79.

15 Ropper AH, Fisher CM, Kleinman M. Pyramidal infarction in the medulla: a cause of pure motor hemiplegia sparing the face. Neurology 1979;29:91-5.

16 Jagiella WM, Sung JH. Bilateral infarction of the medullary pyramids in humans. Neurology 1989;39:21-4.

17 Currier RD, Giles CL, DeJong RN. Some comments on Wallenberg's lateral medullary syndrome. Neurology 1961; 11:778-91.

18 Norrving B, Cronqvist S. Lateral medullary infarction: prognosis in an unselected series. Neurology 1991;41:2448 .

19 Sacco RL, Freddo L, Bello JA, et al. Wallenberg's lateral medullary syndrome. Clinical-magnetic resonance imaging correlations. Arch Neurol 1993;50:609-14.

20 Kim JS, Lee JH, Suh DC, et al. Spectrum of lateral medullary syndrome. Correlation between clinical findings and magnetic resonance imaging in 33 subjects. Stroke 1994;25: magnetic 